\title{
FACTORES DE RIESGO ASOCIADOS A LA RECURRENCIA DE PREECLAMPSIA EN GESTANTES DEL SERVICIO DE ALTO RIESGO OBSTÉTRICO DEL HOSPITAL NACIONAL GUILLERMO ALMENARA IRIGOYEN EN EL 2017 - 2018
}

\author{
Jesús M. Villanueva-Bustamante ${ }^{1,2}$, Jhony A. De La Cruz- Vargas ${ }^{1,2}$, César Raúl Alegría Guerrero ${ }^{3,5, a}$, \\ Pedro M. Arango-Ochante ${ }^{4}$ a
}

\begin{abstract}
RESUMEN
Objetivo: Determinar los factores de riesgo asociados a la recurrencia de preeclampsia en el Hospital Nacional Guillermo Almenara I. (HNGAI) en el 2017-2018. Materiales y métodos: Se trata de un estudio observacional, retrospectivo, analítico tipo casos y controles donde se utilizaron tablas descriptivas y cruzadas para el OR. Resultados: Luego del análisis univariado de los factores de riesgo se vio la mayor prevalencia de gestantes de entre 20 a 35 años con una cifra de $59.4 \%$. Después del análisis bivariado se observó una asociación entre la variable sobrepeso u obesidad y la recurrencia de preeclampsia con un $\mathrm{p}$ valor de 0.031 y un OR de 1.94 (1.05 - 3.56) con IC al 95\%. También se vio asociación entre la ser ama de casa con la recurrencia de preeclampsia con un $p$ valor de 0.030 y un OR de 1.95 (1.06 - 3.58) con un IC de 95\%. Por último, en el análisis multivariado los factores estudiados mantuvieron la significancia la obesidad con un $\mathrm{p}$ valor de 0.036 y un OR ajustado de 1.99 (1.04- 3.79) al IC de $95 \%$ y la variable ocupación un p de 0.023 y un OR ajustado de 2.07 $(1.10-3.90)$ con un IC al 95\%. Conclusiones: Se concluye que las variables sobrepeso u obesidad y ocupación, en este caso ser ama de casa está asociado a recurrencia de preeclampsia.
\end{abstract}

Palabras claves: Factores de riesgo; Obesidad; Recurrencia de preeclampsia (Fuente: DeCS BIREME)

\section{RISK FACTORS ASSOCIATED WITH RECURRENCE OF PREECLAMPSIA IN PREGNANT WOMEN OF THE HIGH RISK OBSTETRIC SERVICE OF GUILLERMO ALMENARA IRIGOYEN NATIONAL HOSPITAL IN 2017-2018}

\begin{abstract}
Objective: To determine the risk factors associated with the recurrence of preeclampsia in the HNGAI of 2017-2018. Materials and methods: This is an observational, retrospective, analytical case-control study where descriptive and cross tables were used for the OR. Results: After the univariate analysis of the risk factors, the highest prevalence of pregnant women between 20 and 35 years of age was seen, with a figure of $59.4 \%$. After bivariate analysis, an association was observed between the variable overweight or obesity and the recurrence of preeclampsia with a p value of 0.031 and an OR of 1.94 (1.05-3.56) with $95 \% \mathrm{Cl}$. An association was also seen between housewife and recurrence of preeclampsia with a $p$ value of 0.030 and an OR of $1.95(1.06-3.58)$ with a $95 \% \mathrm{Cl}$. Finally, in the multivariate analysis, the factors studied maintained obesity with a p value of 0.036 and an adjusted OR of $1.99(1.04-3.79)$ at the $95 \% \mathrm{Cl}$ and the occupation variable a p of 0.023 and an adjusted OR of $2.07(1.10-3.90)$ with a $95 \% \mathrm{Cl}$. Conclusions: It is concluded that the variables overweight or obesity and occupation, in this case being housewives, is associated with recurrence of preeclampsia.
\end{abstract}

Keywords: Risk factors; Obesity; recurrence of preeclampsia (Source: MeSH NLM).

\section{INTRODUCCIÓN}

La preeclampsia patología muy importante en el campo obstétrico, es una de las mayores causas, en el Perú y en el mundo, de la mortalidad materna asociada a complicaciones fetales y neonatales. Por eso es de vital importancia el estudio de los factores de riesgo para conocerlos y poder predecir esta enfermedad, que van a contribuir a disminuir en gran porcentaje mucho de las complicaciones ya mencionadas.

Se han hecho diferentes estudios internacionales, entre los que podemos mencionar el de Mirab Emanuel y Sofía Butt realizado en el periodo 2011-2012 donde se vio que las variables hipertensión crónica, sobrepeso y diabetes están relacionadas a recurrencia preeclampsia. ${ }^{1}$ Van

\footnotetext{
Facultad de Medicina Humana, Universidad Ricardo Palma

Instituto de investigación en Ciencias Biomédicas, Universidad Ricardo Palma

Facultad de Medicina Humana, Universidad Nacional Mayor de San Marcos

Unidad Funcional de investigación INMP

Hospital Guillermo Almenara I.

Médico Ginecoobstetra

Citar como: Villanueva-Bustamante JM, De La Cruz- Vargas JA, Alegria CR, Arango-Ochante PM. Factores de riesgo asociados a la recurrencia de preeclampsia en gestantes del servicio de alto riesgo obstétrico del hospital nacional Guillermo Almenara Irigoyen en el 2017 - 2018. Rev Peru Investig Matern Perinat 2020; 9(2): 26-30

DOI https://doi.org/10.33421/inmp.2020202
}

Recibido: 22-02-20 Aprobado: 30-06-20 
Oostwaard et all. En el 2014 concluyen que el IMC y a la hipertensión crónica son factores asociados a la recurrencia de preeclampsia. $^{2}$ Al siguiente año Van Oostwaard y colaboradores en un metaanálisis concluye entro otros resultados que el porcentaje de recurrencia de preeclampsia fue un $\mathbf{2 0 . 7} \%$ del total, la hipertensión crónica en gestantes después de su primer embarazo donde tuvieron cuadro de preeclampsia tuvo un OR de $3,7 .^{3}$

Arotoma en el 2015 encontró diferentes factores asociados a la presentación de preeclampsia sin tomar en cuenta la recurrencia, como la edad menor de 19 años, primer embarazo, antecedente familiar entre otros. ${ }^{4}$ Thomas P. Bernardes en el 2017 donde el tema de estudio fue acerca del riesgo de recurrencia de preeclampsia con respecto a la máxima presión arterial diastólica y la edad gestacional que hayan tenido en el primer embarazo, realizado en Holanda, observó que la prevalencia de preeclampsia tanto para el primer y segundo embarazo fueron $2.5 \%$ y $0.9 \%$ respectivamente, además también se vio que las mujeres con un antecedente de preeclampsia previa tuvo un riesgo aprox. de $10 \%$ de desarrollar preeclampsia en el siguiente embarazo, otro de los resultados que se obtuvo fue que en las mujeres que en su primer embarazo hayan tenido una $\mathrm{PAD}<80 \mathrm{mmHg}$ tuvieron una recurrencia de preeclampsia del $0.2 \%$ mientras que en las que tuvieron PAD > o igual a $110 \mathrm{mmHg}$ fue de $4.2 \%$. Con respecto a la preeclampsia de inicio tardío y una PAD de 100-109 mmHg la recurrencia de preeclampsia fue de $8.3 \%$, mientras para los que tuvieron PAD > o igual a 110 $\mathrm{mmHg}$ el riesgo de recurrencia fue de $11 \%$. Concluyendo que el riesgo de preeclampsia en un embarazo posterior aumentaba si en el primer embarazo tenía presiones arteriales diastólicas aumentadas asociadas a un inicio tardío de la preeclampsia. ${ }^{5}$

Un estudio realizado por Raúl Alegría Guerrero y Carlos A. Gonzales Medina realizado en el HNGAI nos menciona factores para la predicción de preeclampsia en el primer trimestre como paridad, antecedente de preeclampsia, edad materna, PAM, IMC, índice de pulsatilidad de las arterias uterinas, proteinuria de 24 horas y presencia de alguna comorbilidad, que nos da la posibilidad de una mejor visión en cuanto a la prevención de preeclampsia. ${ }^{6}$

En el Hospital Guillermo Almenara existe una gran demanda de gestantes con esta patología, de los cuales algunos presentan recurrencia de preeclampsia que complican la salud materna y fetal, por lo que nos resulta imperioso hacer un estudio sobre los factores de riesgo o de protección asociadas a esta.

\section{MATERIALES Y MÉTODOS}

Tipo y diseño de investigación: El estudio fue de tipo observacional, analítico, casos y controle de corte retrospectivo para determinar si los factores de riesgo estudiados están asociados a la recurrencia de preeclampsia en el servicio de alto riesgo obstétrico del Hospital Guillermo Almenara, del periodo 2017 a 2018.

Población y muestra: Para el cálculo del tamaño muestral se utilizó el programa estadístico OpenEpi de acceso libre en internet (http://wwww.openepi.com/Menu/ OEMenu.htm) .Se seleccionó la opción de cálculo de tamaño muestral para estudios de tipo caso-control. Para ello se tomó como nivel de confianza al $95 \%$ una potencial estadística del $80 \%$, razón de controles por caso de 2 , porcentaje de controles expuestos de 0.1 y porcentajes de casos con exposición de 0.36 . El cálculo final del tamaño muestral ajustado con la prueba de Fleiss con corrección de continuidad fue de 192, divididos en 64 casos y 128 controles.

Variables e instrumentos: La variable dependiente fue recurrencia de preeclampsia, mientras que las variables independientes fueron edad, tabaquismo, diabetes mellitus, hipertensión arterial, anemia, infección urinaria, número de controles prenatales, ocupación, nivel de instrucción, estado civil, sobrepeso u obesidad, periodo intergenésico corto, los datos de las variables fueron obtenidas de las historias clínicas.

Procesamiento y análisis de datos: Respecto al plan de análisis las variables cualitativas serán descritas mediante frecuencias y porcentajes. Las variables cuantitativas serán analizadas según su normalidad y posteriormente descritas con medidas de tendencia central y dispersión según sea el caso. En el análisis bivariado para determinar las diferencias significativas entre los grupos de categorías, se utilizarán en el caso de variables cualitativas pruebas de chi_cuadrado o test exacto de Fisher, y para variables cuantitativas las pruebas $t$ de student o $U$ de Mann Whitney según sea el caso, con un intervalo de confianza del $95 \%$ y un $p<0.05$ significativo. Posterior a ello, aquellas variables significativas que demuestran diferencias entre si debidas al azar serán analizadas mediante el uso de regresiones logísticas utilizando como medida de asociación ODDS RATIO (OR). Posteriormente aquellas variables que resultaron significativas del análisis bivariado serán analizadas con modelos lineales generalizados con distribución binomial o poisson y función de enlace logístico. El análisis de datos será realizado utilizando el programa estadístico SPSS ver 25.0 con licencia adquirida por el Instituto de Ciencias Biomédicas de la Universidad Ricardo Palma.

Aspectos éticos: En el estudio se hizo la recolección de datos mediante las historias clínicas, para lo cual se hizo un juramento de confidencialidad en el cual se guardó la reserva del caso respecto de información privilegiada a la que pudiera acceder y a no divulgar ni utilizar la información que pudiera ser utilizada en beneficio propio o de terceros con el consiguiente perjuicio a la paciente, ESSALUD y al Estado. 


\section{RESULTADOS}

Con respecto a las características generales, se encontró que la mayoría de la población tenía entre 20 a 35 años teniendo como media 32,4 ; asimismo, el $18,2 \%$ fumaba, un $27,6 \%$ presento diabetes mellitus tipo 2 . Las gestantes con hipertensión arterial fueron de un $12.5 \%$, presentaron anemia un $39.1 \%$ con una media de hemoglobina de 11,8; La población con controles prenatales menor a 6 fue $44.8 \%$, la media fue de 5,59. En cuanto a la ocupación el grupo que corresponde a las amas de casa corresponde a un $42.2 \%$. En el estado civil el grupo predominante fueron las casadas con un $51.6 \%$. El peso normal fue predominante con un porcentaje de $54.7 \%$. Por último, en cuanto al periodo intergenésico menor o igual a 2 años se vio una frecuencia de $20.8 \%$.

Tabla 1. (análisis univariado).

\begin{tabular}{|c|c|c|}
\hline & Frecuencia & Porcentaje \\
\hline \multicolumn{3}{|l|}{ Edad } \\
\hline Menor de 20 años & 10 & $5.2 \%$ \\
\hline Entre 20 y 35 años & 114 & $59.4 \%$ \\
\hline Mayor de 35 años & 68 & 35.4 \\
\hline \multicolumn{3}{|l|}{ Tabaquismo } \\
\hline Si & 35 & $18.2 \%$ \\
\hline No & 157 & $81.8 \%$ \\
\hline \multicolumn{3}{|l|}{ Diabetes mellitus 2} \\
\hline $\mathrm{Si}$ & 53 & $27.6 \%$ \\
\hline No & 139 & $72.4 \%$ \\
\hline \multicolumn{3}{|l|}{ Hipertensión arterial } \\
\hline $\mathrm{Si}$ & 24 & $12.5 \%$ \\
\hline No & 168 & $87.5 \%$ \\
\hline \multicolumn{3}{|l|}{ Anemia } \\
\hline Si & 75 & $39.1 \%$ \\
\hline No & 117 & $60.9 \%$ \\
\hline \multicolumn{3}{|l|}{ Infección urinaria } \\
\hline Si & 50 & $26 \%$ \\
\hline No & 142 & $74 \%$ \\
\hline \multicolumn{3}{|c|}{ Número de controles prenatales } \\
\hline Menor a 6 & 86 & $44.8 \%$ \\
\hline Mayor o igual a 6 & 106 & $55.2 \%$ \\
\hline \multicolumn{3}{|l|}{ Nivel de instrucción } \\
\hline Primaria completa & 15 & $7.8 \%$ \\
\hline Secundaria completa & 89 & $46.4 \%$ \\
\hline Técnico & 28 & $14.6 \%$ \\
\hline Superior & 60 & $31.3 \%$ \\
\hline \multicolumn{3}{|l|}{ Ocupación } \\
\hline Ama de casa & 81 & $42.2 \%$ \\
\hline No ama de casa & 111 & $57.8 \%$ \\
\hline \multicolumn{3}{|l|}{ Estado civil } \\
\hline Soltera & 24 & $12.5 \%$ \\
\hline Casada & 99 & $51.6 \%$ \\
\hline Conviviente & 69 & $35.9 \%$ \\
\hline \multicolumn{3}{|l|}{ Sobrepeso y obesidad } \\
\hline Normal & 105 & $54.7 \%$ \\
\hline Sobrepeso & 33 & $17.2 \%$ \\
\hline Obesidad tipo I & 34 & $17.7 \%$ \\
\hline Obesidad tipo II & 14 & $7.3 \%$ \\
\hline Obesidad tipo III & 6 & $3.1 \%$ \\
\hline \multicolumn{3}{|l|}{ Periodo intergenésico } \\
\hline Menor o igual a 2 años & 40 & $20.8 \%$ \\
\hline Mayor a 2 años & 152 & $79.2 \%$ \\
\hline
\end{tabular}

En el análisis bivariado la recurrencia de preeclampsia se asoció con ser ama de casa con un OR de 1.95 ( 1.063.58) con un IC al $95 \%$ y con tener sobrepeso u obesidad con un OR de 1.94 ( 1.05 - 3.56) con IC al 95\% .La variable edad obtuvo una variación de 0.505 ,el tabaquismo un 0.597 , la diabetes mellitus 0.909 , la hipertensión arterial un 0.165 , la anemia obtuvo un 0.530 ,la infección urinaria un 0.245 , el número de controles prenatales obtuvo 0.051 , el nivel de instrucción obtuvo 0.306 ,el estado civil 1.000 y el periodo intergenésico 0.900 .

Tabla 2. (Análisis bivariado)

\begin{tabular}{|c|c|c|c|c|}
\hline & \multicolumn{2}{|c|}{$\begin{array}{c}\text { Recurrencia } \\
\text { de preeclampsia }\end{array}$} & \multirow{2}{*}{$\begin{array}{c}\text { Valor } \\
p\end{array}$} & \multirow{2}{*}{ OR } \\
\hline & $\mathbf{S i}$ & No & & \\
\hline \multicolumn{5}{|l|}{ Edad } \\
\hline $\begin{array}{c}\text { Menor a } 20 \text { o } \\
\text { mayor a } 35 \text { años }\end{array}$ & $24(30.8 \%)$ & $54(69.2 \%)$ & \multirow{2}{*}{0.505} & \multirow{2}{*}{$\begin{array}{c}0.81 \\
(0.43-1.50)\end{array}$} \\
\hline De 20 a 35 años & $40(35.4 \%)$ & $73(64.6 \%)$ & & \\
\hline \multicolumn{5}{|l|}{ Tabaquismo } \\
\hline Si & $13(37.1 \%)$ & $22(62.9 \%)$ & \multirow{2}{*}{0.597} & \multirow{2}{*}{$\begin{array}{c}1.22 \\
(0.57-2.63)\end{array}$} \\
\hline No & $51(32.5 \%)$ & $106(67.5 \%)$ & & \\
\hline \multicolumn{5}{|c|}{ Diabetes mellitus II } \\
\hline Si & $18(34 \%)$ & $35(66 \%)$ & \multirow{2}{*}{0.909} & \multirow{2}{*}{$\begin{array}{c}1.04 \\
(0.53-2.03)\end{array}$} \\
\hline No & $46(33.1 \%)$ & $93(66.9 \%)$ & & \\
\hline \multicolumn{5}{|c|}{ Hipertensión arterial } \\
\hline $\mathrm{Si}$ & $11(45.8 \%)$ & $13(54.2 \%)$ & \multirow{2}{*}{0.165} & \multirow{2}{*}{$\begin{array}{c}1.83 \\
(0.77-4.36)\end{array}$} \\
\hline No & $53(31.5 \%)$ & $115(68.5 \%)$ & & \\
\hline \multicolumn{5}{|l|}{ Anemia } \\
\hline Si & $23(30.7 \%)$ & $52(69.3 \%)$ & \multirow{2}{*}{0.530} & \multirow{2}{*}{$\begin{array}{c}0.82 \\
(0.44-1.52)\end{array}$} \\
\hline No & $41(35 \%)$ & $76(65 \%)$ & & \\
\hline \multicolumn{5}{|l|}{ Infección urinaria } \\
\hline Si & $20(40 \%)$ & $30(60 \%)$ & \multirow{2}{*}{0.245} & \multirow{2}{*}{$\begin{array}{c}1.48 \\
(0.76-2.89)\end{array}$} \\
\hline No & $44(31 \%)$ & $98(69 \%)$ & & \\
\hline \multicolumn{5}{|c|}{ Número de controles prenatales } \\
\hline Menor a 6 & $35(40.7 \%)$ & $51(59.3 \%)$ & \multirow{2}{*}{0.051} & \multirow{2}{*}{$\begin{array}{c}1.82 \\
(0.99-3.34)\end{array}$} \\
\hline Mayor o igual a 6 & $29(27.4 \%)$ & $77(72.6 \%)$ & & \\
\hline \multicolumn{5}{|l|}{ Ocupación } \\
\hline Ama de casa & $34(42 \%)$ & $47(58 \%)$ & \multirow{2}{*}{0.030} & \multirow{2}{*}{$\begin{array}{c}1.95 \\
(1.06-3.58)\end{array}$} \\
\hline No ama de casa & $30(27 \%)$ & $81(73 \%)$ & & \\
\hline Nivel de instruc & ción & & & \\
\hline Primaria / & & & & \\
\hline $\begin{array}{l}\text { secundaria } \\
\text { completa }\end{array}$ & $38(36.5 \%)$ & $66(63.5 \%)$ & 0.306 & $\begin{array}{c}1.37 \\
(0.74-2.52)\end{array}$ \\
\hline Técnico/Superior & $26(29.5 \%)$ & $62(70.5 \%)$ & & \\
\hline Estado civil & & & & \\
\hline Soltera & $8(33.3 \%)$ & $16(66.7 \%)$ & & 1.00 \\
\hline $\begin{array}{c}\text { Casada/ } \\
\text { conviviente }\end{array}$ & $56(33.3 \%)$ & $112(66.7 \%)$ & 1.000 & $(0.40-2.47)$ \\
\hline Sobrepeso y ob & esidad & & & \\
\hline Si & $36(41.4 \%)$ & $51(58.6 \%)$ & 11 & 1.94 \\
\hline No & $28(26.7 \%)$ & $77(73.3 \%)$ & 0.031 & $(1.05-3.56)$ \\
\hline Periodo interge & nésico & & & \\
\hline $\begin{array}{c}\text { Menor o igual a } \\
2 \text { años }\end{array}$ & $13(32.5 \%)$ & $27(67.5 \%)$ & 0.900 & $\begin{array}{c}0.95 \\
(0.45-2.00)\end{array}$ \\
\hline Mayor a 2 años & $51(33.6 \%)$ & $1016.4 \%)$ & & \\
\hline
\end{tabular}

En el análisis multivariado, se incluyeron las variables sobrepeso/obesidad, ocupación, número de controles prenatales e hipertensión arterial. Se observa que las variables sobrepeso/obesidad y ocupación mantiene su 


\section{SOBREPESO Y OBESIDAD}

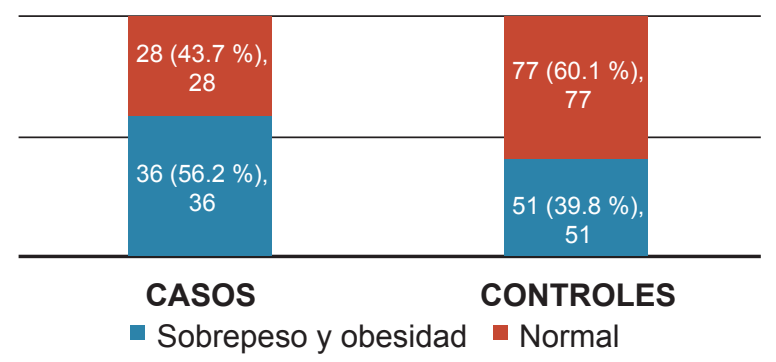

Gráfico 1. Sobrepeso y obesidad.

\section{OCUPACIÓN}

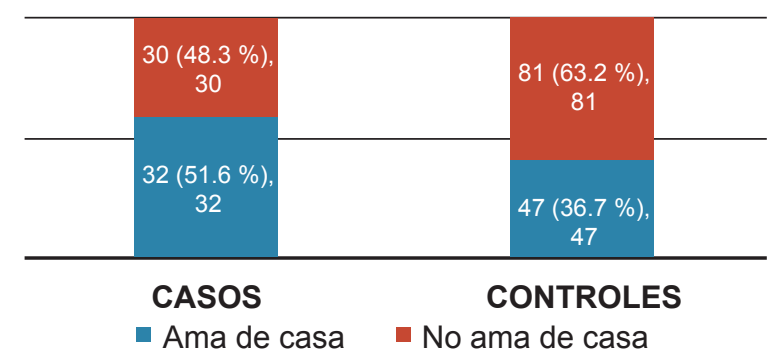

Gráfico 2. Ocupación.

asociación con un valor $p$ de 0.036 y 0.023 respectivamente además el Odds Ratio en ese orden fue de 1.99 (1.04 $3.79)$ y $2.07(1.10-3.90)$ con un IC de $95 \%$. Mientras que la hipertensión arterial y el número de controles prenatales obtuvieron un $p$ valor de 0.303 y 0.53 respectivamente; en el OR ajustado obtienen $1.62(0.64-4.06)$ y 1.86 (0.993.48 ) respectivamente con un IC al $95 \%$.

Tabla 3. Análisis multivariado.

\begin{tabular}{ccc}
\hline & $\begin{array}{c}\text { Variación } \\
\text { p }\end{array}$ & $\begin{array}{c}\text { OR ajustado } \\
\text { (IC 95\%) }\end{array}$ \\
\hline Sobrepeso y obesidad & 0.036 & $1.99(1.04-3.79)$ \\
Ocupación & 0.023 & $2.07(1.10-3.90)$ \\
Número de controles prenatales & 0.053 & $1.86(0.99-3.48)$ \\
Hipertensión arterial & 0.303 & $1.62(0.64-4.06)$ \\
\hline
\end{tabular}

\section{DISCUSIÓN}

Como hemos visto en los antecedentes se tiene noción de los factores de riesgo y de protección en el desarrollo de la preeclampsia, por lo que ahora con este estudio se intenta ver la importancia de estas mismas variables, pero en la recurrencia de la preeclampsia.

El estudio fue realizado en el Hospital Guillermo Almenara con el fin de tener una muestra mucho más amplia y diversa, además de las comorbilidades que presentan los pacientes, en este caso las gestantes.

Consideramos que el estudio toma relevancia desde el punto de vista preventivo ya que sabiendo un poco más de las variables y su asociación o no con la recurrencia de preeclampsia se tomarán medidas con el fin de monitorizar y controlar los factores que están siendo estudiados para poder disminuir la recurrencia de preeclampsia y así las complicaciones maternas perinatales y neonatales.

En el presente estudio la variable sobrepeso u obesidad está asociada a recurrencia de preeclampsia, concordando con el estudio realizado por Van Oostwaard y Col. donde también se observó asociación con la recurrencia de preeclampsia7. Muy en contraste con el estudio hecho por Lien Trana y Col. en el 2019 donde no se encontró diferencias significativas entre los casos y controles para la recurrencia de preeclampsia ${ }^{8}$.

Respecto a la variable ocupación, también está asociada a la recurrencia de preeclampsia, en este caso no se encontraron estudios a nivel nacional ni internacional para poder contrastar con los resultados del estudio. En esta situación se extrapolaron estudios donde el objetivo fue ver las variables asociadas al desarrollo de preeclampsia. Tomando en cuenta esto se hace mención al estudio realizado por Ipiales, Silva y Coedro en el Hospital Enrique C. Sotomayor el 2015 donde se encontró asociación entre la ocupación el ser amas de casa y desarrollo de preeclampsia ${ }^{9}$.

Una característica importante del grupo de las amas de casa es que generalmente van acompañadas de un nivel de instrucción básico que en el estudio abarca a las gestantes con primaria y secundaria completa y además un nivel socioeconómico pobre. Estas variables han sido estudiadas en el desarrollo de preeclampsia por Díaz Villanueva en el año 2016 donde se observó que de las pacientes que presentaban preeclampsia el $70 \%$ pertenecía a un nivel socioeconómico pobre y el $63.3 \%$ solo llegaba a estudios de secundaria ${ }^{10}$. El estudio hecho por Guananga y Coronel en el Hospital de Guayaquil en el periodo 2017 donde se vio también que la clase socioeconómico baja era muy prevalente en pacientes que desarrollaban preeclampsia ${ }^{11}$.

En el presente estudio también tener en cuenta las limitaciones que se presentaron, como el tamaño de la población ya que quizás se necesitaron más casos y controles para un mayor poder estadístico de las variables estudiadas, también la subjetividad a las que están expuestas el llenado de las historias ya que la recolección de datos fue a partir de ellas teniendo fe y objetividad en lo escrito en ellas. En cuanto a la búsqueda de antecedentes los estudios fueron limitados respecto a la recurrencia de preeclampsia.

En conclusión, el sobrepeso u obesidad y el ser ama de casa están asociados a recurrencia de preeclampsia, mientras que las variables edad, tabaquismo, diabetes mellitus, hipertensión arterial, anemia, infección urinaria, número de controles prenatales, nivel de instrucción, estado civil y periodo intergenésico corto no estuvieron asociados a recurrencia de preeclampsia. 
Financiamiento: Autofinanciado.

Conflicto de interés: Los autores declaran no tener algún conflicto de intereses.

\section{REFERENCIAS BIBLIOGRÁFICAS}

1. Mirab Emanuel, Sofia Butt. Frequency and factors leading to recurrent pre-eclampsia.Journal of the Pakistan Medical Association.2015; 65 (11).1173-1177.Disponible en: https:// www.researchgate.net/publication/283791891_Frequency_ and_factors_leading_to_recurrent_pre-eclampsia

2. Miriam F. Van Oostwaard, Josje Langenveld, Ewoud Schuit, Kiki Wigny, Hilde Van Susante, Irene Beune; et al. Prediction of recurrence of hypertensive disorders of pregnancy in the term period, a retrospective cohort study.Pregnancy Hypertension: An International Journal of Women"s Cardiovascular Health.2014. Disponible en: https://www.ncbi. nlm.nih.gov/pubmed/26104605

3. Miriam F. Van Oostwaard; at all. Recurrence of hypertensive disorders of pregnancy: an individual patient data metanalysis. American Journal of Obstetrics and Gynecology, 2015; 212. Disponible en: https://www.ncbi.nlm.nih.gov/ pubmed/25582098.

4. Arotoma Ore Marcelo. Factores de riesgo materno perinatales asociados a preeclampsia, Hospital Víctor Ramos Guardia. Repositorio institucional UNASAM.2019. Disponible en: http://repositorio.unasam.edu.pe/handle/UNASAM/3420

5. Thomas P. Bernardesa, Ben W. Molb, Anita C.J. Ravellic, Paul P. van den Bergd,H. Marike Boezena, Henk Groena. Recurrence risk of preeclampsia in a linked populationbased cohort: Effectsof first pregnancy maximum diastolic blood pressure and gestational age. Rev Pregnancy Hypertension.2017; Vol 15: 32-36.Disponible en: https://www.sciencedirect.com/science/article/abs/pii/ S221077891730452X

6. Raúl Cesar Alegría Guerrero, Carlos A.Gonzales-Medina. Evaluación diagnóstica de un normograma de predicción de preeclampsia. Rev Perú Investig Matern Perinat. 2018 ; 7(2) :21-30.Disponible en: https://investigacionmaternoperinatal. inmp.gob.pe/index.php/rpinmp/article/view/114
7. Miriam F. van Oostwaard, MD; Josje Langenveld, MD, PhD; Ewoud Schuit, MSc, PhD; Dimitri N. M. Papatsonis, MD, PhD; Mark A. Brown, MD, PhD; Romano N. Byaruhanga, MD, PhD; Sohinee Bhattacharya, MD, PhD; Doris M. Campbell, MD, PhD; Lucy C. Chappell, MD, PhD; Francesca Chiaffarino, $\mathrm{ScD}$; Isabella Crippa, MD, PhD; Fabio Facchinetti, MD, PhD; Sergio Ferrazzani, MD, PhD; Enrico Ferrazzi, MD, PhD; Ernesto A. Figueiro'-Filho, MD, PhD; at all. Recurrence of hypertensive disorders of pregnancy : an individual patient data metanalysis.American Journal of Obstetrics and Gynecology . 2015; 212(5):624.e1-17.Disponible en: https:// www.ncbi.nlm.nih.gov/pubmed/25582098

8. Phuong Lien Trana, Pierre-Yves Robillardb,c , Coralie Dumonta , Chloé Schweizera, Asma Omarjeea , Silvia lacobellib, Malik Boukerrou. Recurrent or first preeclampsia in multiparae: A case - control study of singleton pregnancies in Reunion Island. Eur J Obstet Gynecol Reprod Biol. 2019; 240: 80 - 86. Disponible en: https://www.ncbi.nlm.nih.gov/ pubmed/31234061

9. Juan Pablo Ipiales Vásconez, Jacqueline Gabriela Silva Vaca, Alberto Cordero Aroca. Factores de riesgo para preeclampsia en el Hospital Enrique C. Sotomayor. Revista de la Facultad de Ciencias Médicas de la Universidad de Guayaquil.2015; Vol. 18, N 2. Disponible en: http://eluniversitario.edu.ec/ revistas/index.php/RFCM/article/view/61.

10. Díaz Villanueva Joel. Factores de riesgo para preeclampsia en pacientes adolescentes atendida en el Hospital Sergio. E. Bernales en el año 2015. Revista de la Facultad de Medicina Humana de la Universidad Ricardo Palma.2016.Disponible en: http://repositorio.urp.edu.pe/handle/urp/505

11. Álvarez Ponce Vivian, Martos Benites Frank. El sobrepeso y la obesidad como factores de riesgo para preeclampsia. Revista cubana de Obstetricia y Ginecología. 2017; 43(2). Disponible en: http://www.revginecobstetricia.sld.cu/index. php/gin/article/view/208/154

\section{Correspondencia:}

Jesús Villanueva Bustammante

Dirección: Calle San Mateo Asoc. Santa María del Valle Etapa III Mz. C Lt. 14 San Martín de Porres Teléfono: 956293656 